\title{
RCA combined nanoparticle-based optical detection technique for protein microarray: a novel approach
}

\author{
Hsin-Yun Hsu, Yi-You Huang* \\ Institute of Biomedical Engineering, College of Engineering, College of Medicine, National Taiwan University, \\ No. 1, Sec. 1, Jen-Ai Road, Taipei 100, Taiwan, ROC
}

Received 3 June 2003; accepted 14 October 2003

Available online 10 April 2004

\begin{abstract}
Developing a readily available biosensor with excellent performances is the main focus of many research groups. Recently, major breakthroughs in miniaturization of molecular analysis have produced DNA and protein microarrays. The aim of our group is to develop a sensitive technique for analyzing signals on protein microarray by applying the surface plasmon resonance (SPR) method. This new detection technique for specific molecular binding utilizes rolling circles amplification (RCA) post-signal processing method [Nat. Genet. 19 (1998) 225-232] and optical visualization by nanogold particle-labeled molecules on a micro-structured chip surface. By covalent bonding of the RCA primer to the detection antibody guarantees that the linkage between the analyte and the amplified RCA product is maintained during the assay. Experimental results show that RCA has significantly enhanced sensitivity compared to conventional methods. This combination of an easily detectable signal with chip technology should have the potential to become a successful commercial application.
\end{abstract}

(C) 2004 Elsevier B.V. All rights reserved.

Keywords: Nanogold; Rolling circles amplification (RCA); Protein microarrays

\section{Introduction}

Microarrays are two-dimensional patterns consisting of surface-immobilized molecules of defined molecular species. Compared with gene/DNA chips, establishing parallel analysis of protein function using corresponding technologies have been much more elusive (Schweitzer and Kingsmore, 2002). Unlike hybridization, which involves interaction of linear DNA or RNA sequences, protein-protein interactions depend on their folded, three-dimensional protein structures. Maintaining the folded properties of proteins may pose a potential problem in the fabrication of protein microarrays. Furthermore, compare to hybridization reactions, proteins are also prone to nonspecific interactions due to the structure similarity. Another method of detecting the amount of proteins depends on measuring the amount of the corresponding mRNA. However, it is difficult to draw an absolute correlation between abundance of mRNA and the level of its corresponding protein (Gygi et al., 1999), because

\footnotetext{
* Corresponding author. Tel.: +886-2-23222499; fax: +886-2-23940049.

E-mail address: yyhuang@ha.mc.ntu.edu.tw (Y.-Y. Huang).
}

it depends on a number of factors, such as post-translational modifications, proteolysis, and compartmentalization.

Nevertheless, several researches have demonstrated the developments of array-based platforms for studying protein expression. These include the use of membrane-based arrays to screen for DNA, RNA, or protein-binding targets in bacterial expression libraries $(\mathrm{Ge}, 2000)$ and filter-based ELISAs to characterize single-chain antibody libraries (de Wildt et al., 2000). Array formats have also been used to monitor protein-protein interactions, quantitate antibodies (Joos et al., 2000), detect enzyme targets, and analyze interactions between proteins and small molecule drugs (MacBeath and Schreiber, 2000).

Methods currently used to detect specific molecular binding predominantly depend on using optical immunoassay, such as surface plasmon resonance (SPR) (Nelson and Krone, 1997; Rich and Myszka, 2001). SPR system uses excitation with coherent light in attenuated total internal reflection geometry (ATIR) to obtain discrimination between surface-bound and free molecules in solution. Under ATIR, the reflected photons create an electric field on the opposite side of the interface. Similarly, plasmons in the surface film create an electric field called the evanescent field that 
extends into the medium on either side of the film. The equations that describe how electric fields travel through a medium include terms representing the properties of the medium. For light waves, these properties are embodied in the refractive index. The resonance angle is influenced by the refractive index. Furthermore, the resonance angle and the refractive index are both consequences of the equations describing how electric fields interact with a medium. When the analyte concentration at the sensor surface changes, the velocity (the momentum) of the surface plasmons is affected, and the incident light angle at which resonance occurs shifts.

Most of these devices that detect specific molecular binding consist of a defined biological component that provides the required specificity and a transducer that generates the signal. Both components have to be in close contact with each other. Similar to conventional immunoassays, these devices all have a solid phase, which facilitates washing and separation steps and serves as a matrix for the immobilization of the ligand and a labeled component. Specific binding could be detected by the use of tagged molecules. A new approach was to use the colloidal gold as the detection label (Storhoff et al., 1998; Taton et al., 2000; Taton, 2002). First, oligonucleotides with complementary sequences to the target frame are attached to colloidal gold particles. After the arrays are incubated with the solution, the colloids will link with the target ss-DNA, resulting in a color change of the solution. Such a signal can be easily detected without sophisticated and expensive fluorescence equipment. Moreover, by applying a silver enhancement technique, combinatorial DNA or protein arrays can be easily monitored using a conventional flatbed scanner.
The goal of our research group is to develop a sensitive method for analyzing signals on either a DNA or a protein chip (with goat anti-rabbit polyclonal antibody and anti-goat-IgG as the testing model) utilizing the SPR theory. The aim is to develop a new detection technique for the specific molecular binding based on rolling circles amplification (RCA) post-signal processing method (Lizardi et al., 1998; Schweitzer et al., 2000) and optical visualization of nanogold particle-labeled molecules on a micro-structured chip surface (Fig. 1). Covalent bonding of the RCA primer to the detection antibody guarantees that the linkage between the analyte and the amplified RCA product is maintained during the assay. RCA has significantly enhanced sensitivity compared to conventional methods. This combination of an easily detectable signal with chip technology should have the potential to become a successful commercial application.

\section{Antibody-based detection systems combined with RCA}

The RCA system is a novel approach for post-signal amplification of molecular recognition events. There are numerous examples where important biological markers for cancer, infectious disease, or biochemical processes are present in body fluids or tissues at a concentration too low to be detected by conventional immunoassays. Recent advances in the field of low-level antigen detection include the development of stronger fluorochromes and chemiluminescent substrates for use in ELISAs, immunofluorescence-based staining and immunoblotting. Although these techniques can be quite powerful, greater

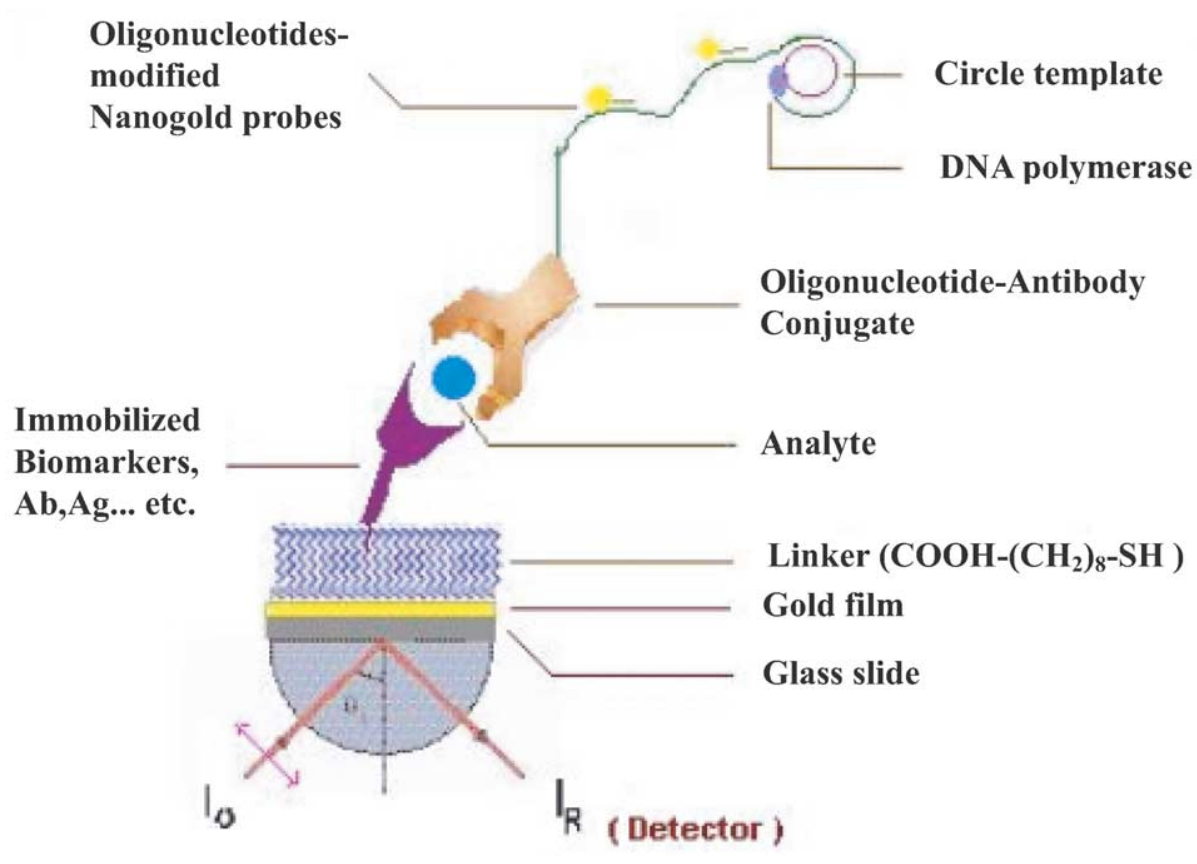

Fig. 1. The RCA combined nanoparticle-based optical detection model. 
sensitivity and specificity are often required, particularly when working with limited amounts of sample material or when antigen density is extremely low. One solution is to use DNA amplification for the detection of antibodies bound to antigen (Ruzicka et al., 1993; Zhou et al., 1993). Multiple antibodies and multiple DNA tags have been used to analyze several antigens simultaneously (Hendrickson et al., 1995). Although immunoPCR was shown to be significantly more sensitive than ELISA, thermal cycling is necessary and product separations by gel electrophoresis have restricted its widespread adoption. Because of the limitation of these techniques, we adapted the recently developed RCA system for the detection of biomolecules, which has previously been used for the detection of antibodies bound to antigen (Schweitzer et al., 2000).

RCA driven by DNA polymerase can replicate circularized oligonucleotide probes with either linear or geometric kinetics under isothermal conditions. Using a single primer, RCA can generate hundreds of tandemly linked copies of the circular template within a short period of time (Fire and $\mathrm{Xu}, 1$ 1995; Khudyakov and Fields, 2003). When the $5^{\prime}$ end of the RCA primer is attached to an antibody, in the presence of circular DNA, DNA polymerase, and nucleotides, the rolling circle reaction takes place and results in a DNA molecule consisting of multiple repeats of the circle DNA sequence that remains attached to the antibody. As a result, by using a single primer, the amplified DNA can be detected in a variety of ways, including direct incorporation of hapten-labeled or fluorescently labeled nucleotides, or by hybridization of fluoro-labeled or enzymatically labeled complementary oligonucleotide probes. In our studies, we choose oligonucleotides-modified nanogold particles as the detection probe. Fig. 2 shows the TEM images of $16 \mathrm{~nm}$ nanogold particles before and after the amplification by the rolling circles amplification, with $\mathrm{P}-5^{\prime}$ CCC TAT ACA CCC AAG CTT CCC ACC GGA ATT $3^{\prime}$ as the circle template and $\mathrm{SH}-5^{\prime} \mathrm{CAC}$ CCA AGC TTC CCA C $3^{\prime}$ (sequences complementary to the RCA products) as the oligonucleotides attached on the nanogold particles. To consider of the nonspecific effect, nanogold with non-complementary sequences had also been examined, and results showed no aggregates (data not shown).
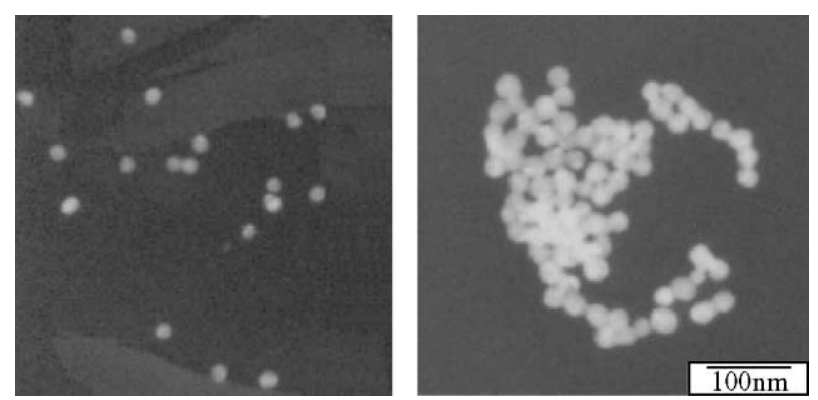

Fig. 2. TEM images of $16 \mathrm{~nm}$ nanoprobes before (left) and after (right) applying the RCA reaction.

\section{Nanogold-based optical detection}

Nanogold-based optical detection can provide a visible range of visualization. By relying on basic optical phenomena, the detection procedure is significantly easier than fluorescence techniques. The stability of the samples is also greatly enhanced. As a result, high light intensities can be applied for detection in contrast to the sensitive fluorescence dyes and their bleaching problem. This advantage can lead to significantly shorter detection time. The increased stability may be useful to provide the possibility of reanalyzing of samples. The influence by the chemical and physical environment on the signal intensity is significantly reduced, resulting in robust measurements. This allow for enhanced reproducibility and comparison between different assays and chips. Another important reason is the potential of miniaturization.

Nanogold particles can also improve the detection signal of SPR (Lyon et al., 1998, 1999; He et al., 2000). Usage of the gold nanoparticle tags leads to a greater increase in angle shift, allowing improvements in sensitivity compared to other non-gold nanoparticle-assisted binding events. The enhanced shift in SPR reflectivity is a combined result of greatly increased surface mass, high dielectric constant of gold particles, and electromagnetic coupling between gold nanoparticles and the gold film. The high signal-to-noise ratio of the particle labels points to the possibility of spot sizes approaching the refraction limit in the sub-micrometer range, compared to the medium micrometer range used in fluorescence chips. It shows that colloidal gold particles can be used for the chip-based detection of specific biomolecular binding. This approach enables the application of optical readout without the need of fluorescence equipment. Nanogold-modified oligonucleotides probes may be regenerated for reuse. These improvements of the detection method provide the means for a new class of chip detection systems.

Important issues, such as characterization of the RCA detection conjugates should show that the binding specificity and the priming efficiency of oligonucleotides are still retained. In a recent study (Peña et al., 2002), the steric effects on the hybridization and enzymatic extension of oligonucleotide-bound nanogold particles had been investigated and the results have shown that linker length and surface coverage play important roles in the reaction. In addition, problems, such as avoiding washing steps, and preventing interference from the sample matrix, and the design of the flow channel within the sensor chip to allow non-diffusion limited mass transport, needs to be solved to reduce assay times.

\section{Conclusion}

An ideal system should combine the advantages of simple easy-use test strips, like simple handling, with the advantages of micro-titer plate assays or automated 
laboratory equipment, for high reproducibility, high sensitivity, and quantitative measurements. The field of nanoparticle amplified-SPR is still very much in its infancy. This short communication demonstrates the possibility of performing RCA with nanoparticle-amplified SPR in biomolecules detection and its potential for future applications in ultra-sensitive DNA/protein microarray analysis.

\section{References}

Fire, A., Xu, S.-Q., 1995. Rolling replication of short DNA circles. Proc. Natl. Acad. Sci. U.S.A. 92, 4641-4645.

Ge, H., 2000. UPA, a universal protein array system for quantitative detection of protein-protein, protein-DNA, protein-RNA and protein-ligand interactions. Nucleic. Acids Res. 28, e3.

Gygi, S.P., Rochon, Y., Franza, B.R., Aebersold, R., 1999. Correlation between protein and mRNA abundance in yeast. Mol. Cell. Biol. 19, 1720-1730.

He, L., Musick, M.D., Nicewarner, S.R., Salinas, F.G., Benkovic, S.J., Natan, M.J., Keating, C.D., 2000. Colloidal Au-enhanced surface plasmon resonance for ultrasensitive detection of DNA hybridization. J. Am. Chem. Soc. 122, 9071-9077.

Hendrickson, E.R., Hatfield, T.M., Joerger, R.D., Majarian, W.R., Ebersole, R.C., 1995. High sensitivity multianalyte immunoassay using covalent DNA-labeled antibodies and polymerase chain reaction. Nucleic Acids Res. 23, 522-529.

Joos, T.O., Schrenk, M., Hopfl, P., Kroger, K., Chowdhury, U., Stoll, D., Schorner, D., Durr, M., Herick, K., Rupp, S., Sohn, K., Hammerle, H., 2000. A microarray enzyme-linked immunosorbent assay for autoimmune diagnostics. Electrophoresis 21, 2641-2650.

Khudyakov, Y.E., Fields, H.A. 2003. Artificial DNA: Methods and Applications, CRC Press.

Lizardi, P.M., Huang, X., Zhu, Z., Bray-Ward, P., Thomas, D.C., Ward, D.C., 1998. Mutation detection and single-molecule counting using isothermal rolling-circle amplification. Nat. Genet. 19, 225232 .
Lyon, L.A., Musick, M.D., Natan, M.J., 1998. Colloidal Au-enhanced surface plasmon resonance immunosensing. Anal. Chem. 70, 51775183.

Lyon, L.A., Peña, D.J., Natan, M.J., 1999. Surface plasmon resonance of Au colloid-modified Au films: particle size dependence. J. Phys. Chem. B 103, 5826-5831.

MacBeath, G., Schreiber, S.L., 2000. Printing proteins as microarrays for high-throughput function determination. Science 289, 1760-1763.

Nelson, R.W., Krone, J.R., 1997. Surface plasmon resonance biomolecular interaction analysis mass spectrometry. Part 1. Chip-based analysis. Anal. Chem. 69, 4363-4368.

Peña, S.R.N., Raina, S., Goodrich, G.P., Fedoroff, N.V., Keating, C.D., 2002. Hybridization and enzymatic extension of Au nanoparticle-bound oligonucleotides. J. Am. Chem. Soc. 124, 73147323.

Rich, R.L., Myszka, D.G., 2001. BIACORE J: a new platform for routine biomolecular interaction analysis. J. Mol. Recognit. 14, 223-228.

Ruzicka, V., Marz, W., Russ, A., Gross, W., 1993. Immuno-PCR with a commercially available avidin system. Science. 260, 698-699.

Schweitzer, B., Wiltshire, S., Lambert, J., O’Malley, S., Kukanskis, K., Zhu, Z., Kingsmore, S.F., Lizardi, P.M., Ward, D.C., 2000. Immunoassays with rolling circle DNA amplification: a versatile platform for ultrasensitive antigen detection. Proc. Natl. Acad. Sci. U.S.A. 97, 10113-10119.

Schweitzer, B., Kingsmore, S.F., 2002. Measuring proteins on microarrays. Curr. Opin. Biotechnol. 13, 14-19.

Storhoff, J.J., Elghanian, R., Mucic, R.C., Mirkin, C.A., Letsinger, R.L., 1998. One-pot colorimetric differentiation of polynucleotides with single base imperfections using gold nanoparticle probes. J. Am. Chem. Soc. 120, 1959-1964.

Taton, T.A., Mirkin, C.A., Letsinger, R.L., 2000. Scanometric DNA array detection with nanoparticle probes. Science 289, 1757-1760.

Taton, T.A., 2002. Nanostructures as tailored biological probes. Trends Biotechnol. 20, 277-279.

de Wildt, R.M., Mundy, C.R., Gorick, B.D., Tomlinson, I.M., 2000. Antibody arrays for high-throughput screening of antibody-antigen interactions. Nat. Biotechnol. 18, 989-994.

Zhou, H.R., Fisher, J., Papas, T.S., 1993. Universal immuno-PCR for ultra-sensitive target protein detection. Nucleic Acids Res. 21, 60386039. 